\title{
Analysis of pH Dependent Luminescence of an Europium Complex
}

\author{
Fang XIE ${ }^{a}$, Hao LIANG $^{b,}$, , Gui-Qiang DIAO ${ }^{c}$, Fu-Sheng LUO ${ }^{d}$
}

Department of Chemical Engineering, Huizhou University, Guangdong 516007, China

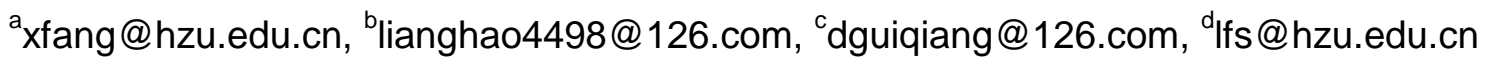

${ }^{*}$ Corresponding author

Keywords: PH, Luminescence, Europium, Complex.

Abstract. The $\mathrm{pH}$ dependence of the photoluminescence of an europium complex in aqueous solution was measured and investigated. The fluorescence emission spectra showed $\mathrm{pH}$ sensitivity. The analysis of the relative intensity ratio (R) of ${ }^{5} \mathrm{D}_{0} \rightarrow{ }^{7} \mathrm{~F}_{2}$ to ${ }^{5} \mathrm{D}_{0} \rightarrow{ }^{7} \mathrm{~F}_{1}$ transition and Judd-Ofelt experimental intensity parameters $\Omega_{2}$ indicated that the local environment around the $\mathrm{Eu}^{3+}$ and the asymmetry of the Eu complex have been influenced by the $\mathrm{pH}$ value of the aqueous solution.

\section{Introduction}

The design of compounds that facilitate sensing is an active area of research [1]. Lanthanides possess intrinsic luminescence that originates from $\mathrm{f}-\mathrm{f}$ electron transitions in the $4 \mathrm{fn}$ shell of the [Xe]5s25p6 configuration and offer unique properties for optical sensing. The unique luminescence properties of $\mathrm{Eu}(\mathrm{III})$ complexes, such as long luminescence lifetimes, sharp and intense emission bands, make them especially useful for the design of luminescent sensing materials[2 4].

In the fields of chemistry, biochemistry and environmental science, the detection and quantification of $\mathrm{pH}$ value is very important. In this work, the $\mathrm{pH}$ dependence of the photoluminescence of an europium complex in solution was measured and investigated by Judd-Ofelt theory. The result will provide useful information for designing lanthanide complex-based luminescent sensors.

\section{Experimental}

The molecular structure of the europium complex with cyclen derivative is shown in Fig. 1 It was synthesized according to the procedure reported by Gunnlaugsson et al [5].Elemental analysis calculated for the europium complex $\left(\mathrm{EuC}_{35} \mathrm{H}_{51} \mathrm{~N}_{10} \mathrm{O}_{7} \mathrm{~F}_{3} \mathrm{~S}\right)$ : C,43.57\%; H,5.33\%; N,14.52\%; S,3.32\%; found: C, 43.49\%; H, 5.29\%; N, 14.61\%; S,3.30\%.

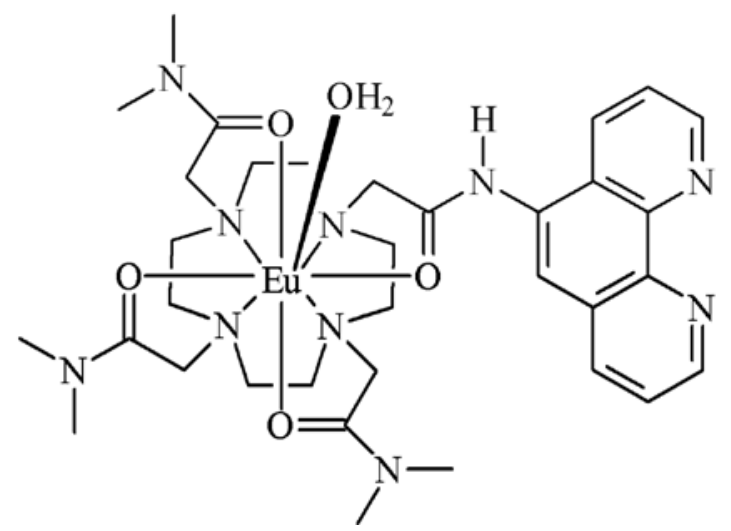

Fig. 1 Molecular structure of europium complex with cyclen derivative

Elemental analyses were performed using a Perkin-Elmer 2400CHN elemental analyzer. The fluorescence emission spectra of the europium complex were recorded on a Shimadzu RF-5301PC spectrofluorophotometer. The $\mathrm{pH}$ dependence of the $\mathrm{Eu}(\mathrm{III})$ emission was evaluated in $\mathrm{H} 2 \mathrm{O}$ in the presence of $0.1 \mathrm{M}$ tetramethylammonium perchlorate to maintain constant ionic strength. 


\section{Results and Discussion}

\section{PH Dependence of Fluorescence Emission Spectra of Eu Complex in Solution}

The pH dependence of fluorescence emission spectra of Eu complex in solution are shown in Fig.2.

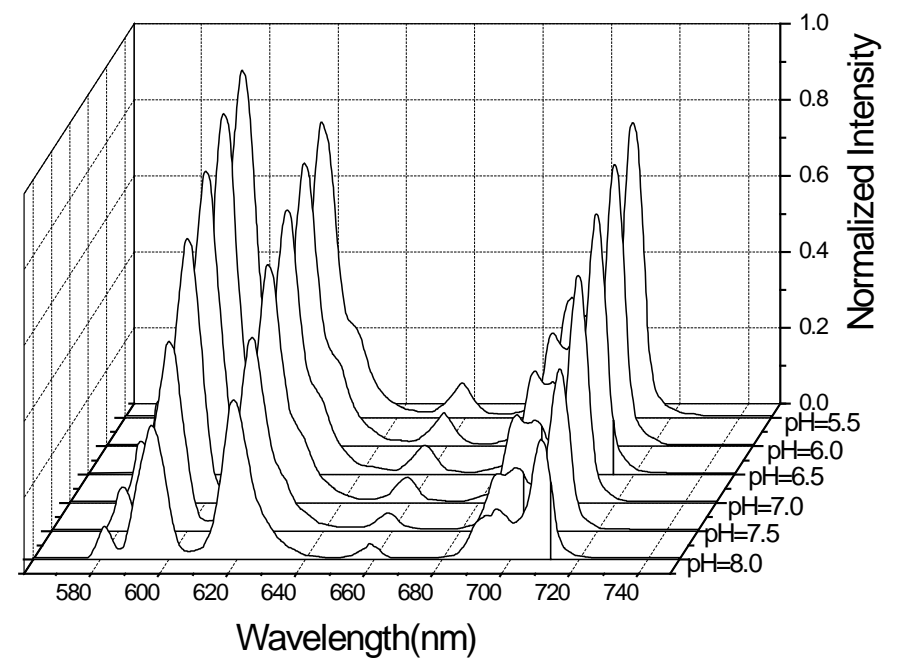

Fig.2 pH dependence of fluorescence emission spectra of Eu complex in solution

The emission spectrum was recorded form $550 \mathrm{~nm}$ to $750 \mathrm{~nm}$ under the excitation at $266 \mathrm{~nm}$. It can be found five emission peaks corresponding to ${ }^{5} \mathrm{D}_{0} \rightarrow{ }^{7} \mathrm{~F}_{0,1,2,3,4}$ can be clearly distinguished for Eu complex in solution. The presence of only one ${ }^{5} \mathrm{D}_{0} \rightarrow{ }^{7} \mathrm{~F}_{0}$ line indicates that the $\mathrm{Eu}^{3+}$ ion occupies only a single site and a single chemical environment exists around it [6].

It can be found in Fig.2 that the emission intensity decreased with the increase in $\mathrm{pH}$. Through an intramolecular ligand to rare earth ion energy transfer process, the metastable state of the rare earth ion can be populated by pumping ligand absorption bands, which are much stronger than those of the rare earth. In this case, the population of the Eu(III) excited state (5D0) is achieved by the ligands antenna effect. Both the amide and the nitrogens of the phen ligand are sensitive to protonation, thus the emission intensity decreased significantly with the increase in $\mathrm{pH}$.

\section{Relative Intensity Ratio}

The ${ }^{5} \mathrm{D}_{0} \rightarrow{ }^{7} \mathrm{~F}_{1}$ transition is usually used as a reference because it is allowed by magnetic dipole and its intensity is independent of the environment. Because the variation of the relative intensity ratio (R) of $5 \mathrm{D} 0 \rightarrow 7 \mathrm{~F} 2$ to $5 \mathrm{D} 0 \rightarrow 7 \mathrm{~F} 1$ transition is very sensitive to the structural change in the vicinity of Eu3+ ions, it can be used to reflect the local structure and asymmetry in the vicinity of europium ions [7]. Fig.3 shows the $\mathrm{R}$ ratio values of Eu complex in solution with different $\mathrm{pH}$.

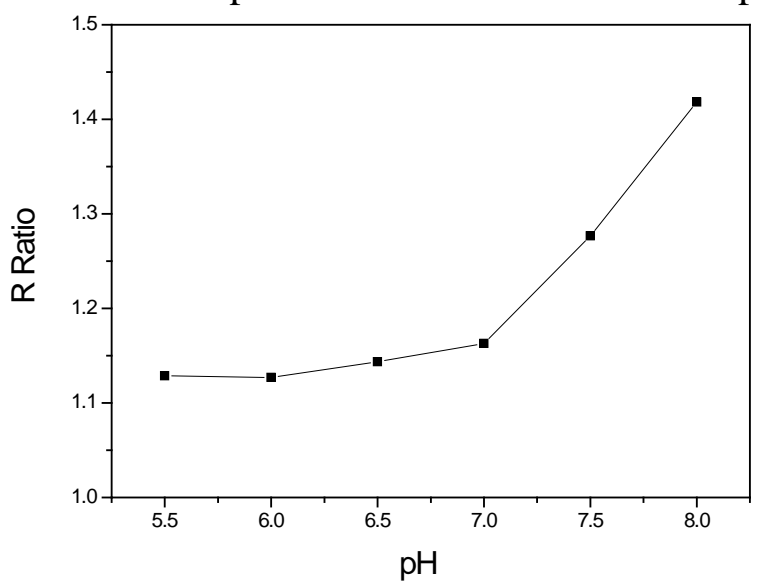

Fig.3 R ratio values of Eu complex in solution with different $\mathrm{pH}$ 
In Fig.3, it can be found that the values of $\mathrm{R}$ ratio showed significant change with the increasing of $\mathrm{pH}$. This indicates that there is also change in the local environment and asymmetry in the vicinity of europium ion when the $\mathrm{pH}$ is increased. According to the chemical structure of Eu complex showed in Fig.1, the ligands of the Eu complex are protonation sensitive. With the increasing of $\mathrm{pH}$, the local environment around the Eu3+ has been changed, which leads to the significant variation of $\mathrm{R}$ ratio.

\section{Judd-Ofelt Analysis}

According to Judd-Ofelt theory, the calculated oscillator strength for an induced electric dipole transition from the ground state to an excited state is given by Eq.1 [8]:

$$
f=\frac{8 \pi^{2} m c v}{3 h(2 J+1)} \frac{\left(n^{2}+2\right)^{2}}{9 n} \sum_{\lambda=2,4,6} \Omega_{\lambda}\left(\Psi J\left\|U^{\lambda}\right\| \Psi^{\prime} J^{\prime}\right)^{2}
$$

where $n$ is refractive index of the medium, $h$ is Planck's constant, $J$ is the total angular momentum of the ground state, $v$ is the wave number of the transition, $\Omega_{\lambda}(\lambda=2,4,6)$ are Judd-Ofelt intensity parameters and $\left\|U^{\lambda}\right\|^{2}(\lambda=2,4,6)$ are the doubly reduced matrix elements evaluated in the intermediate coupling approximation for a transition $\Psi J \rightarrow \Psi^{\prime} J^{\prime}$. The transition intensity $f$ depends on the $\left\|U^{\lambda}\right\|^{2}$ values between $\Psi J$ and $\Psi^{\prime} J^{\prime}$ manifold. Due to selection rules and the unique nature of transition intensities for $\mathrm{Eu}^{3+}$ ion, any one of the $\left\|U^{\lambda}\right\|^{2}$ parameters decide the intensities of the transitions since the remaining two are zero. Thus, $\Omega_{\lambda}(\lambda=2,4,6)$ parameters can be evaluated independently from the emission transitions of ${ }^{5} \mathrm{D}_{0} \rightarrow{ }^{7} \mathrm{~F}_{2},{ }^{5} \mathrm{D}_{0} \rightarrow{ }^{7} \mathrm{~F}_{4}$ and ${ }^{5} \mathrm{D}_{0} \rightarrow{ }^{7} \mathrm{~F}_{6}$, respectively [9]. The intensity of the ${ }^{5} \mathrm{D}_{0} \rightarrow{ }^{7} \mathrm{~F}_{1}$ fluorescent transition is found to be host independent whereas that of the ${ }^{5} \mathrm{D}_{0} \rightarrow{ }^{7} \mathrm{~F}_{\mathrm{J}}$ transition depends on the $\Omega_{\lambda}$ parameters. This characteristic helps one to evaluate the $\Omega_{\lambda}$ parameters simply by the ratio of the intensities of the ${ }^{5} \mathrm{D}_{0} \rightarrow{ }^{7} \mathrm{~F}_{2,4,6}$ transitions to the intensity of ${ }^{5} \mathrm{D}_{0} \rightarrow{ }^{7} \mathrm{~F}_{1}$ transition as Eq.2 [8].

$$
\int I_{J} d v / \int I_{1} d v=A_{J} / A_{1}=\frac{e^{2}}{S_{m d 1}} \frac{v_{J}^{3}}{v_{1}^{3}} \frac{n\left(n^{2}+2\right)^{2}}{9 n^{3}} \sum \Omega_{\lambda}\left\|U^{\lambda}\right\|^{2}
$$

where $S_{m d 1}$ refers to the strength of the magnetic dipole line strength of the ${ }^{5} \mathrm{D}_{0} \rightarrow{ }^{7} \mathrm{~F}_{1}$ transition in absorption.

The obtained values for parameter $\Omega_{2}$ and $\Omega_{4}$ of Eu complex in solution with different $\mathrm{pH}$ are listed in Table I . The $\Omega_{6}$ intensity parameter was not determined because the ${ }^{5} \mathrm{D}_{0} \rightarrow{ }^{7} \mathrm{~F}_{6}$ transition could not be experimentally detected.

Tab.1 $\Omega_{2}, \Omega_{4}$ parameters of of Eu complex in solution with different $\mathrm{pH}$

\begin{tabular}{ccc}
\hline $\mathrm{pH}$ & $\Omega_{2}$ & $\Omega_{4}$ \\
\hline 5.5 & $1.98 \times 10^{-20} \mathrm{~cm}^{2}$ & $7.89 \times 10^{-20} \mathrm{~cm}^{2}$ \\
6.0 & $1.99 \times 10^{-20} \mathrm{~cm}^{2}$ & $7.97 \times 10^{-20} \mathrm{~cm}^{2}$ \\
6.5 & $2.01 \times 10^{-20} \mathrm{~cm}^{2}$ & $7.84 \times 10^{-20} \mathrm{~cm}^{2}$ \\
7.0 & $2.05 \times 10^{-20} \mathrm{~cm}^{2}$ & $7.84 \times 10^{-20} \mathrm{~cm}^{2}$ \\
7.5 & $2.25 \times 10^{-20} \mathrm{~cm}^{2}$ & $7.67 \times 10^{-20} \mathrm{~cm}^{2}$ \\
8.0 & $2.50 \times 10^{-20} \mathrm{~cm}^{2}$ & $7.97 \times 10^{-20} \mathrm{~cm}^{2}$ \\
\hline
\end{tabular}

According to previous studies, $\Omega_{2}$ is a useful parameter, because it is sensitive to the local symmetry of the ligand field and bond covalency [10]. The value of $\Omega_{2}$ increases as the local symmetry of the ligand field decreases and as the bond covalency increases. It can be conclude that the decrease of the $\mathrm{pH}$ also increased the asymmetry of the Eu complex. The $\Omega_{4}$ parameter has been related together to bulk properties of the lanthanide based hosts, but there is no theoretical prediction for this sensibility to macroscopic properties [11]. 


\section{Summary}

In conclusion, the $\mathrm{pH}$ dependence of the photoluminescence of an europium complex in aqueous solution was measured and investigated. The Judd-Ofelt phenomenological parameters, $\Omega_{2}$ and $\Omega_{4}$ were obtained from the fluorescence emission spectrum. The analysis of the relative intensity ratio (R) and $\Omega_{2}$ indicated that the local environment around the $\mathrm{Eu}^{3+}$ and the asymmetry of the Eu complex have been influenced by the $\mathrm{pH}$ value of the aqueous solution.

\section{Acknowledgement}

This research was financially supported by the Natural Science Foundation of Guangdong Province, China (2014A030313642).

\section{References}

[1] J.Hammell, L.Buttarazzi, C.Huang, J.R.Morrow, Eu(III) Complexes as Anion-Responsive Luminescent Sensors and Paramagnetic Chemical Exchange Saturation Transfer Agents, Inorg. Chem. 50 (2011) 4857-4867.

[2] M.I.J.Stich, L.H.Fischer, O.S.Wolfbeis, Multiple Fluorescent Chemical Sensing and Imaging, Chem. Soc. Rev. 39 (2010) 3102-3114.

[3] B.C.Barja, P.F.Aramend, Luminescent Eu(III) Hybrid Materials for Sensor Applications, Photochem. Photobiol. Sci. 7 (2008) 1391-1399.

[4] K.Binnemans, Lanthanide-based luminescent hybrid mterials, Chem. Rev. 2009, Vol.109:4283

[5] T.Gunnlaugsson, J.P.Leonard, K.S.chal, A.J.Harte, pH Responsive Eu(III)-Phenanthroline Supramolecular Conjugate: Novel“Off-On-Off” Luminescent Signaling in the Physiological pH Range J. Am. Chem. Soc. 125 (2003) 12062-12063.

[6] H.F.Brito, O.L.Malta, L.R.Souza, J.F.S.Menezes, C.A.A.Carvalho, Luminescence of the Films of Europium (III) with Thenoyltrifluoroacetonate and Macrocyclics, J.Non-Cryst.Solids. 247 (1999) 129-133.

[7] H.Liang, Z.Zheng, Q.Zhang, H.Ming, B.Chen, J.Xu, H.Zhao, Radiative Properties of Eu(DBM)3Phen-doped Poly(methyl methacrylate), J. Mater. Res. 18 (2003) 1895-1899.

[8] P.Babu, C.K.Jayasankar, Optical Spectroscopy of $\mathrm{Eu}^{3+}$ ions in Lithium Borate and Lithium Fluoroborate Glasses, Physica B 279 (2000) 262-281.

[9] H.Liang, F.Xie, H.Zhou, G.Zhong, B.Chen, Luminescence Comparison of Europium Complexes with Different Conjugation Length $\beta$-diketone in Polymer, Optoelectron. Adv. Mater. 4 (2010) 1477-1480.

[10] A.G.Souza Filho, J.Mendes Filho, F.E.A.Melo, M.C.C.Custodio, R.Lebullenger, A.C.Hernandes, Optical Properties of $\mathrm{Sm}^{3+}$ Doped Lead Fluoroborate Glasses, J. Phys. Chem. Solids. 61 (2000) 1535-1542.

[11] H.Liang, F.Xie, X.Ren, Y.Chen, B.Chen, F.Guo, Temperature Dependent Luminescence of a Europium Complex Incorporated in Poly(methylmethacrylate), Spectrochimica Acta Part A 116 (2013) 317-320. 